\title{
DESAFÍOS DE LA LEY 7600 ANTE LAS NUEVAS TENDENCIAS DE LA EDUCACIÓN INCLUSIVA
}

\author{
Tania Elena Moreira Mora \\ Asesora de evaluación del Ministerio de Educación Pública \\ San José, Costa Rica
}

Recibido 9-II-2008 • Aceptado 11/III/2008 • Corregido 29/IV/2008

\begin{abstract}
Resumen: En este artículo interesa profundizar en la trayectoria histórica y en las perspectivas teóricas de la educación inclusiva, así como proponer algunos desafios de la Ley 7600 ante el contexto actual de la educación para todos, a partir de una indagación bibliográfica sobre la evolución de este paradigma.

Se presentan las principales perspectivas contemporáneas como la diversidad, normalización, derecho humano, los valores y la interculturalidad que consolidan el surgimiento de un prisma inclusivo. En consonancia con tales enfoques se proponen algunos desafíos de la Ley 7600 sobre la Igualdad de las personas con discapacidad de 1996 en el contexto educativo de Costa Rica. Estos retos se orientaron hacia el desarrollo de proyectos educativos, el trabajo cooperativo, la consolidación de valores, la capacitación docente y los recursos humanos y materiales con una intención en común: contribuir con el cumplimiento de los objetivos de la ley.
\end{abstract}

Palabras clave: Educación, inclusión, paradigma, Ley 7600 .

\section{Introducción}

Durante los últimos decenios ha emanado una sucesión de legislaciones, declaraciones internacionales, debates, investigaciones y publicaciones concernientes a los temas de equidad, igualdad de oportunidades, diversidad, interculturalidad, inclusión/exclusión y derechos humanos, tanto en el ámbito educativo como social, enfocados no solamente en las personas con alguna discapacidad o deficiencia, sino también a quienes son excluidos por sus diferencias sociales, culturales, religiosas, étnicas, de género y tendencias sexuales entre otros; especialmente en la era de la globalización y la informatización que ha provocado una deconstrucción y deshumanización de los sujetos, que se visibiliza en los estereotipos étnicos, la segregación, la desintegración familiar y social, la pobreza, el desempleo y la explotación del recurso humano y natural, entre otros.

Costa Rica ha sido una sociedad caracterizada por su complejidad étnica y cultural derivada de la convivencia de grupos heterogéneos; así como por el crecimiento de inmigrantes de otras latitudes latinoamericanas, aunadas 


\begin{abstract}
In this article they analyze the historical trajectory and the theoretical perspective of the inclusive education and set out some challenges of Law 7600 in the present context of the education for all, from a bibliographical investigation on the evolution of this paradigm. The main contemporary perspective appears like: the diversity, the normalization, the human right, the values and the cross-cultural view, that consolidate the sprouting of a inclusive approach. As a result of those positions challenges of Law 7600 are indicated on the equality of the people with disability in the educative context of Costa Rica. These challenges were oriented to the development of educative projects, the cooperative work, the consolidation of values, the educational qualification and the dowry of human and material resources with an intention in common: to contribute with the exact fulfillment of the objectives of the law.
\end{abstract}

Key words: Education, inclusion, paradigm, Law 7600 . a las diferencias regionales, sociales y económicas, que han llegado a conformar un prisma humano. No obstante, ante la inmersión en la globalización económica y cultural, predominan los enfoques curriculares centrados en los elementos de la cultura considerada oficial desde una visión academicista y normalizada, sustentada más en la competitividad individual que en la pluralidad social y cultural. Como lo enfatiza Yanes (2008) hoy en día la educación se desarrolla en función del poder político, económico social y cultural, reproduciendo constantemente el orden social existente y distribuyendo los bienes culturales de acuerdo con los intereses de los grupos dominantes.

Entonces, dentro de este entorno de desigualdades sociales y humanas, la educación inclusiva se ha orientado hacia la equidad de oportunidades y de derechos dentro de una sociedad que acepte como principio ético la diversidad, ligada a la noción de justicia social, donde cada miembro pueda contribuir y participar en la construcción de una comunidad más solidaria y democrática. Por ello, el propósito de este artículo es profundizar en los antecedentes históricos y en las perspectivas actuales de la educación inclusiva, así como proponer algunos desafíos de la Ley 7600 ante una realidad multicultural y heterogénea como la costarricense.

\section{Retrospección de la educación especial}

La noción y enfoques de la educación especial han evolucionado desde una perspectiva demonológica hasta la inclusividad. Tal evolución responde a los condicionantes históricos, culturales, científicos y educativos enmarcados principalmente en tres grandes momentos o etapas.

La primera es conocida como la prehistoria de la educación especial o primeras experiencias, según Palmero (2000), caracterizada por un enfoque pasivo al 
considerar la discapacidad o anormalidad como fruto de causas ajenas al ser humano. De esta forma, con algunas excepciones, en las sociedades primitivas y en la Edad Media se atribuía a los malos espíritus la causa de la conducta anormal. Tal concepción demonológica generó una actitud radical de rechazo, aceptación resignada de la deficiencia, la curación espiritual mediante la magia o la súplica a los dioses y/o la eliminación del deficiente. En este contexto apenas tenía cabida la educación, ya que la sociedad se preocupaba más por ocultarlos o eliminarlos que por educarlos. En el siglo XIX se gesta la segunda etapa conocida como la era del progreso y las instituciones, en la cual surge un interés por el estudio de la deficiencia mental y la atención hacia las personas con discapacidad; lo que se traduce en importantes avances en su comprensión, descripción, clasificación e identificación, diferenciando entre enfermedad y deficiencia mental, en la mejora de los servicios, en la creación y el desarrollo de la educación especial y la psiquiatría. Es en esta época cuando aparecen los nuevos tratamientos y modelos educativos caracterizados por llevarse a cabo en instituciones y ambientes separados de la educación ordinaria. Ya en el siglo XX esta concepción segregadora es superada por una actitud integradora y de normalización, por un nuevo enfoque y práctica de la educación especial, basada en el desarrollo de la integración y la capacidad de las personas, cuyo rasgo principal es el optimismo pedagógico, aunado a cambios legislativos, que han provocado un lento pero progresivo cambio conceptual y metodológico de la educación especial (Palmero, 2000).

Durante este siglo XX se desarrollaron dos grandes vertientes teóricas de la educación especial, las biológicas y las psicológicas. De las primeras derivan los criterios patológicos de la anormalidad y de las segundas la psicometría, con su fundamento estadístico en la desviación a la norma de la medición de las poblaciones; tales temas abordados con un fundamento positivista de la ciencia y la técnica (García, 2003; Guajardo, 1999). Con ambas teorías se promueve un salto muy importante en el enfoque de la educación especial, pero no de tipo paradigmático. Más bien, este cambio se genera por la presencia de otros actores que no eran los científicos, ni los técnicos, ni los profesionales; sino los usuarios o consumidores de los servicios de educación especial, con los padres de familia, quienes realizaron demandas que evidenciaban el carácter segregado de las prácticas educativas especiales, basadas en teorías de la justicia, la democracia y los derechos humanos (Bautista, 1993; García, 2003; Guajardo, 1999).

Además de lo expuesto anteriormente, otros argumentos que sustentaron el movimiento de la integración fueron el escaso fundamento científico para mantener separados a los niños y jóvenes con discapacidad en centros de educación especiales, más al comprobarse los beneficios culturales y educativos a partir de la convivencia dentro de ambientes menos restrictivos y discriminatorios. También las pruebas psicométricas utilizadas para el diagnóstico resultaban culturalmente tendenciosas, lo que podía acarrear diagnósticos inadecuados y, por consiguiente, a un etiquetamiento y ubicación escolar poco apropiada. Igual importancia tuvieron los argumentos relacionados con la rentabilidad de las inversiones, que hacen más aconsejable la integración de estas minorías en el sistema regular (Bautista, 1993).

En correspondencia con los condicionantes anteriores, han prevalecido por varias décadas dos perspectivas de la educación especial. La individual que asume los problemas de aprendizaje, el déficit o la falta de adaptación a la escuela como una característica inherente al estudiante, por tanto, los profesionales deben diagnosticar, evaluar, clasificar y proveer los servicios que cubran esas necesidades individuales como una respuesta racional al problema de inadaptación (Echeita y Sandoval, 2002; García, 2003; Peralta, 2001). Desde tal 
concepción los servicios y ayudas se brindan a grupos homogéneos de alumnos con similares deficiencias, básicamente por un criterio de rentabilidad, lo que ha llevado a la segregación en centros especializados y a una política de compensación hasta más allá del siglo XX (Echeita y Sandoval, 2002). Esto ha causado el uso de prácticas simplistas y descripciones inadecuadas de la conducta de los estudiantes (García, 2003).

A raíz de sus fuertes críticas, apuntan Echeita y Sandoval (2002) emerge la perspectiva contextual o social centrada en la eliminación de las barreras creadas por unas organizaciones sociales con escasa o nula consideración hacia las personas con necesidades educativas especiales (NEE) y que más bien tienden a excluir esta población de la participación en actividades sociales generales. Además señalan que la inclusión o exclusión no son estados o situaciones definitivas, sino un proceso en que ambos extremos están en continua tensión, de forma que el avance hacia uno solo se produce por la reducción significativa del otro; por tanto, la participación no depende únicamente de que los alumnos se adapten al centro, sino también de que el sistema educativo en su conjunto se transforme y adopte una perspectiva social interactiva. En la actualidad coexisten ambas tendencias, mas no cabe duda del dominio de la perspectiva individual para explicar los comportamientos extraños o diferentes a la "norma", cuyos planteamientos se orientan más hacia una solución parcial o superficial de las carencias o déficit de los estudiantes.

En el contexto educativo costarricense se entiende por educación especial como el "conjunto de apoyos y servicios a disposición de los alumnos con necesidades educativas especiales, ya sea que los requieran temporal o permanentemente" (Ministerio de Educación Pública [MEP], 1998, p. 5). Tal concepción es congruente con las tendencias actuales de integrar a los estudiantes con NEE en ambientes menos restrictivos con los apoyos y servicios necesarios para optimizar su proceso de inclusión en el sistema educativo regular y ofrecerles una educación de calidad, pero aún falta mucho camino por recorrer y barreras por superar para llegar a esta meta.

\section{Hacia la perspectiva de la inclusividad}

Los preludios de la inclusividad se sitúan en la coyuntura social y política de los años sesenta y setenta del siglo XX con el surgimiento de movimientos contra la discriminación, especialmente racial, feminista, a grupos minoritarios y con discapacidad. Ulteriormente con las diversas declaraciones de las Naciones Unidas, que culminaron en el Programa de Acción Mundial para Personas con Discapacidad en 1982 (Fernández, 2003). No obstante, es a partir de 1990 con la Conferencia de la UNESCO en Jomtien (Tailandia) sobre las desigualdades en el ejercicio de los derechos humanos, especialmente en el ámbito de la educación especial, cuando surge el germen de la idea de inclusión (Parrilla, 2002). Posteriormente se promulgaron las Normas Uniformes sobre la Igualdad de Oportunidades para las Personas con Discapacidad (1993), aprobadas por la Asamblea General de las Naciones Unidas que instan a los estados a garantizar que las personas con discapacidad formen parte integrante del sistema educativo general (Fernández, 2003). Luego en 1994 se realiza la Conferencia Mundial de Salamanca sobre Necesidades Educativas Especiales. Acceso y Calidad, patrocinada por la UNESCO, en la cual se consolidan las bases para un cambio de perspectiva de la educación especial y la idea de promover sistemas educativos con una orientación inclusiva, donde se adscribieron 88 países y 25 organizaciones internacionales (Echeita y Sandoval, 2002; Fernández, 2003; Parrilla, 2002).

Desde este momento se inicia un fuerte movimiento mundial en la búsqueda 
de mayores condiciones de equidad en sociedades cada vez más segregadoras; ocasionado en parte por las asimetrías en las relaciones sociales y económicas del proceso de globalización, centrado en la competitividad individual y el poder económico, que ha llevado a la exclusión y marginación de grandes sectores sociales. En esta coyuntura América Latina no ha sido la excepción, la marginación de grandes sectores de la población, la exclusión de grupos indígenas, la xenofobia, el predominio de estereotipos étnicos y la dominación cultural e ideológica de Estados Unidos y otras grandes potencias económicas y políticas del mundo han causado la hegemonía de un discurso etnocéntrico y un modelo anglosajón que han agravado las asimetrías sociales y económicas. Para Braslavsky (2006) este orden económico parece ser fuertemente mono cultural e intercultural al mismo tiempo, pero de hecho, termina por promover fuertemente la imagen de una sociedad mono cultural, con elementos predominantemente anglosajones y con una fuerte influencia de la cultura norteamericana.

Dentro de esta coyuntura la educación especial también evoluciona hacia una perspectiva de inclusividad. Desde un primer estadio conocido como la negación del derecho a la educación y de oportunidades vitales, de hecho o de derecho, de aquellos grupos no pertenecientes a la población meta, acompañada además por la discriminación social (García, 2003; Parrilla, 2002). A un segundo momento, década de los noventa, con el reconocimiento del derecho a la educación diferenciada según grupos en condiciones jerarquizadas al incorporarse en un sistema educativo dual con un tronco común pero con otro paralelo para las respuestas especiales, que lleva a plantear a la cultura dominante como la superior y a calificar cualquier desviación como un rasgo de inferioridad (Parrilla, 2002).

Luego a la fase de las reformas integradoras dentro de un contexto político y cultural de discriminación como respuesta inicial a las necesidades humanitarias de la sociedad, especialmente en Estados Unidos con la población negra y grupos minoritarios y no con las necesidades de las personas con discapacidad (Peralta, 2001). En tales reformas se proponen una serie de cambios en los sistemas educativos tendientes a corregir las fuertes desigualdades causadas por la segregación con una dirección más asimilacionista, es decir, salen de las escuelas específicas para incorporarse en las ordinarias, produciéndose una integración física pero no real, que no garantiza el derecho a recibir respuestas a las necesidades desde condiciones de equidad ante la sociedad (García, 2003; Parrilla, 2002). Esta integración se ha reducido básicamente a problemas de ubicación, pero sin atacar los mecanismos de exclusión (García, 2003). Finalmente surge el estadio de las reformas inclusivas tendientes a una profunda transformación del centro educativo que implica una reestructuración política que dé respuesta a las demandas de la igualdad de oportunidades hasta el desarrollo de un modelo capaz de incluir las diferencias dentro de un marco organizativo flexible (García, 2003; Parrilla, 2002).

Con estas dos últimas perspectivas, integración e inclusión, ha surgido la duda si existe una diferencia sustantiva entre ambas, o más bien, es un asunto de novedad conceptual. Por un lado, Díaz (2003) resalta que no solo es una modificación conceptual, sino de actitud ante la vida, una opción ética; pues se cambia la idea de integrar a los excluidos por la de crear una comunidad escolar en la que todos se apoyan mutuamente. Mientras que, por otro lado, Peralta (2001) plantea que la oposición integración e inclusión parece más un asunto de proposición de conceptos novedosos, una mera declaración de intenciones o de identificación de las dificultades de la puesta en práctica del nuevo concepto; con lo cual, una vez detectadas estas dificultades, se volvería a presentar otro nuevo término; es decir, hacer cambios para que todo siga igual. 
En esta transitividad de la educación especial hacia la inclusividad ha cobrado relevancia la noción la diversidad. En general, este concepto se ha enfocado hacia la heterogeneidad y complejidad de la naturaleza humana desde diversos puntos de vista: ideológico, cultural, educativo, histórico, étnico y capacidades, entre muchos otros. No obstante, Aguado (2000) propone tres dimensiones para comprender este concepto dentro de un contexto general. El primero se enfoca hacia la necesidad de redefinir la visión de la diversidad, pues los modelos de déficit entienden las diferencias como alejamientos e inconsistencias en relación con los modelos ideales, donde lo diferente se valora negativamente y se aspira a igualar, surgen los programas compensatorios e integrales; por lo que en una nueva concepción se debe enfatizar el valor de la diversidad, evitar las implicaciones negativas y la comparación con supuestos patrones. La segunda dimensión apunta hacia la modulación de una cultura escolar inclusiva con respecto a las diferentes manifestaciones de la diversidad sustentada en una visión filosófica más amplia y crítica de multiculturalismo que favorezca: una educación equitativa en igualdad de oportunidades, cambios curriculares y pedagógicos, integración de los contenidos multiculturales, igualdad de experiencias eficientes y potenciales, así como la superación de actitudes y valores racistas. Finalmente, para Aguado (2000) la tercera dimensión concierne a la promoción de prácticas inclusivas que garanticen el éxito escolar y eviten la discriminación en el tratamiento educativo basadas en procedimientos de diagnóstico y evaluación que tomen en cuenta las diferencias individuales, culturales, sociales y psicológicas de los estudiantes, las cuales no están tradicionalmente asociadas al aprendizaje de los estudiantes, pero condicionan las expectativas de éxito académico.

En suma, la inclusividad se ha circunscrito en tres ámbitos, uno relativo al concepto mismo de educación inclusiva como constructo aglutinador de una nueva visión y ampliada para la educación escolar; el referido a las condiciones educativas que conducen a unos centros a ser más eficaces en cuanto a dar respuestas equitativas a la diversidad de alumnos que aprenden y que se conecta con los procesos o ciclos de mejo$r a$ a través de los cuales se implementan los cambios prioritarios que los centros desean realizar y, en tercer lugar, el vinculado a una perspectiva sociocrítica en relación con los estudiantes en desventaja educativa, que se visualiza a través del concepto de barreras para el aprendizaje y la participación, las que lejos de ser realidades sustantivas de los propios estudiantes son más bien el resultado de diferentes barreras, situadas en diversos planos o niveles, que inhiben o dificultan las posibilidades de aprendizaje de los estudiantes calificados tradicionalmente como especiales (Sandoval, López, Miquel, Durán, Giné y Echeita, 2002).

En definitiva, la evolución conceptual y filosófica de la educación especial hacia la inclusividad ha sido lenta y cargada de ambivalencias. Además parece que en Costa Rica se ha desarrollado más la vertiente empírica que la epistemológica; es decir, los docentes se han preocupado más por cumplir con las directrices técnicas, curriculares y políticas; así como por aplicar una variedad de estrategias didácticas para atender la diversidad de necesidades de sus estudiantes, pero no han asumido la inclusividad como una nueva visión del mundo, como "una opción de vida en la que cada persona es valorada como ser individual" (Díaz, 2003, p. 192).

En Costa Rica el proceso de inclusión de la población con necesidades educativas especiales se fortaleció con la promulgación de la Ley 7600 sobre la Igualdad de Oportunidades para las personas con Discapacidad, vigente desde el 29 de mayo de 1996. Esta ley se emplaza en el tercer estadio evolutivo de la inclusividad (las reformas integradoras), caracterizado por la integración de los estudiantes con necesidades educativas especiales en los centros 
educativos, con los servicios de apoyo y adecuaciones tendientes a la equiparación de oportunidades y a garantizar iguales condiciones de acceso y participación. Concretamente en el artículo 14 se declara que el Estado garantizará el acceso oportuno a la educación, independientemente de la discapacidad, desde la estimulación temprana hasta la educación superior. Asimismo, en el artículo 18 se establece que podrán recibir su educación en el sistema educativo regular, con los servicios de apoyo necesarios y aquellos que no puedan satisfacer sus necesidades en las aulas regulares, contarán con servicios apropiados para su respectivo desarrollo y bienestar, incluyendo los de centros de enseñanza especial (Costa Rica, 1996). Conforme con esta ley los estudiantes con NEE se integran al sistema regular con los servicios de apoyo requeridos y las adecuaciones curriculares necesarias para garantizar una igualdad de oportunidades educativas y una educación de calidad.

\section{Perspectivas actuales de la educación inclusiva}

Si se tiene como premisa que el ser humano por su misma naturaleza biológica, cultural y psicológica es heterogéneo, entonces todos somos diferentes desde esta óptica. Por tal razón, en esta época post modernista la tendencia es reconocer precisamente el valor de la diversidad desde el paradigma de la inclusividad.

En este sentido, la diversidad es un rasgo inherente y constitutivo de todo grupo humano. La explicitación de esta característica, conjuntamente con la posibilidad de enriquecerse a través de la diferencia, son los dos pilares de la educación en y para la diversidad. Por ello, se emplea un nuevo discurso considerando las semejanzas y no las diferencias de los estudiantes, es decir, "la tendencia es normalizar tanto como sea posible las condiciones de vida y la escolaridad de los alumnos con necesidades educativas especiales" (Devalle y Vega, 1999, p. 29).

Dentro de esta perspectiva, apuntan las autoras que la escuela es el espacio preferencial para desarrollar actitudes individuales y sociales que generen cambios significativos en los sujetos. "La escuela integradora, inclusiva o comprensiva está sustentada en una pedagogía que se opone a la selección jerárquica y propone una voluntad explícita de desarrollar a los alumnos en un contexto escolar respetuoso de la diversidad personal y colectiva" (Devalle y Vega, 1999, p.103). No obstante, es necesario apuntar que el concepto mismo de diversidad ha sido ambivalente en nuestro contexto educativo, por un lado, se definen categorías para clasificar las diferencias, verbigracia problemas de aprendizaje, trastorno por déficit de atención con hiperactividad y autismo por nombrar algunos pocos; más este etiquetamiento connota de por sí una marca negativa que anula su singularidad. Mientras que, desde otra perspectiva más crítica, la diversidad se concibe como una fuente de enriquecimiento social y cultural dentro de un contexto sin taxonomías.

Sobre esta ambigüedad, apunta Contreras (2002) los diversos o los diferentes son "los otros", los que no son iguales a "nosotros"; entonces este nosotros se convierte en la normalidad de la igualdad deseada y los otros son concebidos bajo categorías que los engloban en colectivos de pertenencia, es decir, el otro, el diferente pertenece a un colectivo de diferencias/deficiencias: inmigrante o retraso mental. De este modo añade el autor, la diferencia/deficiencia se define como a-normalidad del individuo por su adscripción a categorías patológicas o de extrañeza social, pero no como categorías patológicas que den cuenta del marco social o institucional en el que aparecen las nonormalidades problemáticas.

Las raíces de esta visión de la diversidad se encuentran en los principios filosóficos de la normalización y la integración, surgidos alrededor de los años setenta, que impulsaron el desarrollo educativo de 
los estudiantes con necesidades educativas especiales dentro de un ambiente normal. Particularmente, se buscaba que la oferta educativa se adaptara a las particularidades de cada uno, reconociéndoles los mismos derechos y brindándoles los servicios necesarios para desarrollar sus posibilidades y vivir una vida semejante a la de cualquier otra; por tanto, la integración era una y se daba cuando los estudiantes participaban en un modelo único y general, que contemplaba las diferencias en un espacio común (Bautista, 1993; Peralta, 2001).

Tanto el principio filosófico de normalización como de integración demandan una igualdad en los aprendizajes entre los estudiantes con ventaja y quienes se encuentran en situaciones de desventaja, no puede ser para unos más conocimientos de idiomas, de informática o de ciencias; para los segundos, lo mínimo, lo básico o lo imprescindible para salir del paso; por ello, más que reducción o eliminación de objetivos, se debe recurrir a estrategias y métodos que permitan el enriquecimiento y la aceleración de sus aprendizajes (Echeita y Sandoval, 2002), o bien, “ipor qué no pensar que la igualdad podría consistir simplemente en no impedir a nadie el acceso a la educación, pero tampoco nada para facilitárselo?" (Fernández, 2002, p. 57).

Este punto de vista de la normalización ha sido polémico y cuestionado por su trasfondo ideológico, ya que se fundamenta en la dicotomía anormalidad/normalidad, mas qué se entiende por anormal. La anormalidad tiende a acentuar la diferencia percibida como deficiencia, como solo carencia o dificultad, como negatividad y no como otra posibilidad, otra forma de ser y sentir con derecho a la existencia (Contreras, 2002). No obstante, si se asumen los roles sociales desempeñados por los sujetos con necesidades educativas especiales (NEE) como valiosos en sí mismos y no por comparación con los otros; como una valoración de la diferencia y no a la adecuación de lo que es norma en la sociedad puede rescatarse un sentido positivo de la normalización, empero el concepto de normalización ha sido utilizado para minimizar las diferencias, antes que para integrarlas y tratar a las personas con discapacidad como si fueran normales, siendo una nueva forma de opresión que les niega a ser diferentes (García, 2003).

Posteriormente surge la visión de inclusividad, que supone dos procesos interrelacionados, uno "incrementar la participación de los alumnos en la cultura y el currículo de las comunidades y escuelas ordinarias y el proceso de reducir la exclusión de los alumnos de las comunidades y culturas normales" (Parrilla, 2002, p. 18). Este planteamiento es de suma relevancia porque la inclusión no es únicamente en el plano espacial, sino también en las relaciones intersubjetivas intra y extra escolar; por ello es una necesidad recurrir a diversas estrategias de socialización y concienciación para generar un ambiente de respeto, solidaridad y participación entre todos los miembros del grupo, la institución y la comunidad, de tal forma que cualquier acción discriminatoria sea eliminada.

Esta perspectiva de la inclusión también concierne a un asunto de los derechos humanos, que según Parrilla (2002), desde el derecho específico defendido en el principio de normalización se pasa al marco amplio de la Declaración de los Derechos Humanos. La inclusión se plantea entonces como un derecho básico de los estudiantes con discapacidad a escolarizarse en la escuela ordinaria, así la exclusión, desde una perspectiva ética, es una amenaza potencial equivalente a la opresión social por motivos de pertenencia a grupos minoritarios, étnicos, de género o de clase social (Echeita y Sandoval, 2002; Parrilla, 2002). Por ello cada país debe diseñar un sistema escolar adaptable a las necesidades de todos los niños creando escuelas inclusivas (Fernández, 2003). Obviamente, esta perspectiva se vincula con el derecho a vivir con dignidad y a ser respetado en una sociedad sin ningún tipo de barrera ni discriminación. 
Igualmente, ha cobrado importancia el fortalecimiento de valores como el respeto, la dignidad y la solidaridad hacia cualquier ser humano, sin distingos de ninguna clase. Desde una vertiente pragmática la educación inclusiva entonces es definida como "una actitud o sistema de valores y creencias y no una acción ni un conjunto de acciones" (Pérez y Prieto, 1999, p. 74). Más que un conjunto de leyes y normas, es básico consolidar un sistema de valores centrados en la naturaleza humana y no en meras acciones.

Con esta visión de inclusión también se busca consolidar el enfoque intercultural en los centros educativos a través del desarrollo de competencias, tanto en los docentes como en los estudiantes, en tres dimensiones: los conocimientos sobre los valores culturales propios y de los otros, la visión del mundo y normas sociales, las habilidades o destrezas y finalmente las creencias y actitudes sobre los grupos minoritarios del interlocutor o mediador cultural, complementados por los valores que forman parte de la sociedad y de los diferentes grupos sociales que la conforman (Malik, 2002; Sanz, 2001). Específicamente, las actitudes se refieren a las cualidades y la aceptación de otras culturas igualmente válidas a la nuestra; a los conocimientos de los grupos sociales, su identidad, sus producciones y sus costumbres y finalmente las habilidades relacionadas con la interpretación y comparación de las diversas perspectivas, hechos, ideas o documentos de otras culturas y con el aprendizaje e interacción para adquirir nuevos conocimientos acerca de las otras culturas y la destreza de practicarlas en situaciones reales y concretas de comunicación e interacción (Malik, 2002). En definitiva la conjunción de este trinomio: creencias, conocimientos y habilidades es fundamental para lograr un cambio paradigmático hacia la inclusividad y reciprocidad cultural en nuestras aulas y consecuentemente en nuestras sociedades.

En síntesis, estas disyuntivas y ambigüedades son complejas, pues se conjuga, por un lado, de acuerdo con Fernández (2002), un planteamiento positivo de incorporar una población segregada y desnaturalizada a ambientes menos restrictivos donde pueden compartir entre pares; pero, por el otro, se le exige su adaptación a un modelo normativo, que les niega su derecho a ser diferentes y menoscaba su propia identidad. Entonces las políticas educativas deben buscar difíciles equilibrios entre igualdad y diversidad, identidad social e identidad singular, justicia y eficacia, interculturalismo y reconocimiento, libertad y responsabilidad. Probablemente para lograr este balance se requiera desarrollar políticas efectivas de descentralización y de autonomía de las escuelas, considerándose cambios en los estándares curriculares (Rivero, 2005).

En la dimensión social, también es necesario transpolar la inclusividad de las paredes institucionales para llegar a construir una sociedad igualmente inclusiva, pues no es suficiente una educación para todos en igualdad de oportunidades y derechos, si al incorporarse el joven/adulto a la sociedad se encuentra con barreras que le impiden su desarrollo profesional y humano. En este sentido, Richardson y Word (2000) destacan las principales características de una sociedad inclusiva. La primera consiste en inclusión social en sí misma, referida a una plena participación en el ámbito político, económico y cultural de todos sus miembros. La segunda compete a la igualdad de oportunidades referidas a un trato imparcial en el trabajo, oportunidades, comercio y servicios distribuidos en la sociedad. Otro componente es la resolución pacífica de los conflictos enfocada al respeto de las leyes, a diferencia de la violencia y el acoso y finalmente una actitud de mente abierta, de mutuo respeto, reconocimiento y generosidad entre las diferentes comunidades. En definitiva, con la interacción de estas cuatro características se puede llegar a consolidar una sociedad más democrática y equitativa, lo cual depende no de la "buena voluntad" de las autoridades políticas, sino 
del accionar de todos los ciudadanos, quienes deben manifestar sus disconformidades y luchar por el disfrute de sus derechos.

En suma, esta concepción de educación inclusiva se fundamenta en el reconocimiento de la diversidad, la defensa de los derechos humanos, el respeto a la diferencia, la dignificación del ser humano y la participación social. Esta visión implica una ruptura de la concepción tradicional del mundo, creencias, valores y percepciones, así como una concienciación social y política de la necesidad de este cambio paradigmático para mirar, escuchar, estimar y dignificar las singularidades.

\section{Desafíos de la Ley 7600}

Con esta ley se ha dado un paso importante en la inclusión de los estudiantes con NEE en el sistema educativo costarricense y después de 10 años de vigencia es oportuno replantearse nuevos retos con el propósito de alcanzar una transformación paradigmática, de tal forma que la inclusión signifique un aporte teórico e ideológico a esta reforma integradora, actualmente sustentada en diversas normativas y procedimientos, pero sin modificar la visión tradicional de la educación costarricense. Como lo expone Cánovas (2005), en un mundo de cambios acelerados la educación ya no puede sustentarse en los viejos paradigmas de verdades absolutas, donde se justificaban los papeles tradicionales de maestros que hablan y alumnos que escuchan en contextos institucionales aislados y conservadores de un legado social implícito de la "función de educar". En consonancia con esta aseveración, Aguado (2000) agrega que los actuales sistemas educativos no son capaces, en la mayoría de los casos, de introducir cambios que vayan más allá de medidas aisladas. Tal afirmación la sustenta en el hecho de que la institución educativa refleja las relaciones sociales y se identifica con los procedimientos homogeneizadores que desconocen las diferencias culturales, también en que el discurso democrático en torno a la diversidad no se enlaza de manera autocrítica con cuestiones de justicia social e igualdad y, entre otros hechos, en que las prácticas de atención a la diversidad no se enlazan con el programa curricular.

Entonces para lograr este salto paradigmático es necesario responder a las nuevas necesidades y a los cambios generacionales, culturales e históricos desde una perspectiva más amplia de justicia social y equidad. En este sentido Irving, Barker, Parker-Jenkins y Hartas (2000) apuntan que se requiere adoptar un amplio concepto de justicia que contribuya a la fundación de una sociedad más justa y dinámica que satisfaga las necesidades individuales y sociales de todos los ciudadanos.

El sistema educativo costarricense no escapa de tales contradicciones, más con el establecimiento de la Ley 7600 sobre la Igualdad de las personas con discapacidad de 1996. En aquel momento y en la actualidad los docentes de primaria como de secundaria no estaban capacitados para atender las necesidades educativas especiales (NEE) de sus estudiantes; verbigracia, en un grupo de 40 o más alumnos se puede encontrar uno con problemas de aprendizaje, otro con alguna deficiencia física $\mathrm{y}$ algún(os) con el trastorno por déficit de atención con hiperactividad y si sumamos las diferencias propias de los estratos sociales y las culturales; obviamente los docentes no pueden brindar una atención apropiada a tantas necesidades y se sienten agobiados por el volumen de trabajo pues deben, en algunos casos, realizar planeamientos curriculares adicionales, así como diferentes tipos de pruebas, entre otras tareas. Esta situación es reafirmada por Peralta (2001, p. 10), quien manifiesta que si la inclusión supone un equilibrio entre el entorno menos restrictivo y la satisfacción de las necesidades educativas, "resulta difícil defender en la práctica que un mismo entorno físico pueda dar respuesta acorde con la diferencia de cada alumno". Ante este entorno de contradicciones y limitaciones 
que ciñe la Ley 7600, se proponen algunos desafíos afines a las perspectivas actuales de la educación inclusiva.

Un primer desafío es la realización de proyectos educativos dentro de la comunidad para crear una red social orientada a la sensibilización del valor de la diversidad y a dar respuestas eficaces a los problemas actuales del centro educativo; donde participen miembros de todos los grupos minoritarios, así como de entidades oficiales y privadas, con dos intenciones: una que los estudiantes con NEE puedan demostrar sus capacidades fuera del ámbito escolar; la otra, una mayor inserción de la comunidad dentro del quehacer institucional. La inclusión no es solo un modelo educativo, también debe ser social, laboral y familiar; en fin, debe ser una nueva visión del mundo.

El asunto ético está explícito en la ley, mas aún prevalecen actitudes discriminatorias hacia esta población con NEE, por tanto, es necesaria la vivencia e interiorización de valores centrados en la igualdad y justicia social dentro de un modelo integral de sociabilidad democrática que los reconozca como ciudadanos con derechos y esencialmente como personas con una identidad, sentimientos y valores. A través de diversas técnicas de socialización, incluso con actividades extracurriculares enfocadas hacia la resolución de conflictos, se puede estimular una convivencia defendible en los principios y valores humanos y sociales, así como una actitud positiva para afrontar sus diferencias; de este modo se asentarán las bases de un futuro ciudadano democrático y ético.

Asimismo es fundamental desarrollar el trabajo en equipo y cooperativo, lo que implica, de manera paulatina, que los estudiantes con NEE se integren en equipos heterogéneos y flexibles para favorecer un aprendizaje entre iguales en un ambiente de cooperativismo, respeto y solidaridad que, a su vez, contribuirá a reconocer las fortalezas y necesidades individuales y colectivas. Igualmente significa un trabajo en equipo de los docentes, tanto en la planificación curricular como en las estrategias pedagógicas y evaluativas, para optimizar una labor cooperativa que favorezcan acciones más coordinadas y de apoyo profesional entre los docentes de las diferentes asignaturas y así superar las medidas aisladas y transitorias que, además de ser agobiantes, no logran impactar en el proceso formativo de todos los estudiantes.

En estrecha relación con lo anterior, es una prioridad la definición de un proyecto curricular integral en el nivel institucional orientado hacia la diversidad que involucre a todos los responsables del proceso educativo y trascienda el devenir político y administrativo. Este proyecto debe sustentarse en las metodologías y técnicas más pertinentes a las necesidades y heterogeneidad de los estudiantes, acordes con las circunstancias del entorno, en la articulación con los contenidos de los programas de estudio y en la flexibilidad para adaptarse de manera adecuada a las políticas generales y no ser un obstáculo para las innovaciones o cambios coyunturales ulteriores. El éxito de cualquier proyecto dependerá del nivel de sensibilidad de toda la comunidad educativa y de la labor conjunta para alcanzar las metas: educar en la diversidad, respetar las diferencias, fortalecer la solidaridad social y humana y, especialmente, acrecentar el éxito académico y personal de todos los estudiantes.

Otro desafío fundamental es la formación y capacitación del personal docente en temas de discapacidad, interculturalidad y diversidad a través de jornadas de charlas, asesorías y talleres, así como la divulgación de información reciente (investigaciones, modelos educativos, experiencias pedagógicas exitosas, entre otros) que les permita desarrollar adecuadamente el proceso de atención a la diversidad, comprender su papel de mediadores, disponer de herramientas didácticas y evaluativas pertinentes y actuar asertivamente ante las particularidades de su grupo. Asimismo, estas experiencias de capacitación deben 
adecuarse a las necesidades de los docentes, conjugar la teoría con la práctica educativa y ser una respuesta real a sus inquietudes pedagógicas; de lo contrario, serán meras actividades programadas, ajenas a su realidad inmediata, sin ningún valor e impacto en la labor cotidiana del docente.

Igualmente es necesario proveer de los recursos materiales y humanos mínimos a los centros educativos para que los docentes puedan satisfacer de la mejor manera las necesidades de todos sus estudiantes dentro de un entorno que los apoye, motive y ofrezca los materiales indispensables. Mas ello requiere de la articulación de los recursos institucionales y comunitarios disponibles con la programación curricular y el conjunto de actividades del quehacer educativo cotidiano para aprovecharlos de manera eficaz y así evitar la subutilización o el derroche.

A modo de síntesis, retomo el planteamiento de Manucci (2004), quien afirma que las personas no son simples espectadores de una puesta en escena predeterminada; sino que a través del intercambio de subjetividades, la multiplicidad de significados, la interdiscursividad, el conjunto de vivencias y expectativas a lo largo del tiempo y el espacio; así como el azar y la incertidumbre se constituyen en factores interpretativos y constructivos de la realidad. Este es el desafío más importante de la Ley 7600, romper con las estructuras pedagógicas tradicionales para construir un conocimiento holístico y articulado con las prácticas sociales, el pensamiento, los valores, la cotidianidad y las actitudes dentro de un contexto histórico, ecológico y democrático.

\section{Conclusiones}

En la primera parte de esta investigación se hace una retrospección de la educación especial para describir los avances científicos, sociales y educativos más destacables en el desarrollo de una práctica educativa más integral, desde la visión demonológica de la Edad Media hasta las perspectivas actuales de la inclusividad.

En un inicio dominaba una concepción primitiva de la discapacidad que generó actitudes de rechazo, resignación y misticismo. Luego en el siglo XIX un interés científico por las discapacidades mentales incidió en el desarrollo de nuevos tratamientos médicos y educativos. Hasta llegar al siglo $\mathrm{XX}$ donde se transciende la concepción de segregación por la integración e inclusión mediante nuevos modelos pedagógicos y la promulgación de diversas legislaciones. A pesar de la vigencia de acuerdos internacionales y de los avances científicos y educativos, esta trayectoria no ha sido uniforme, como tampoco las decisiones gubernamentales dirigidas hacia los cambios sociales y educativos para la inclusión de quienes históricamente han sido marginados de una educación con calidad y equidad.

Las raíces del modelo inclusivo se asientan en la coyuntura política y social de los años sesenta y setenta del siglo $\mathrm{XX}$ con el surgimiento de los movimientos contra la discriminación, aunado a las diversas declaraciones internacionales, reformas integradoras e inclusivas tendientes a garantizar los derechos de las personas que han sido segregadas por sus condiciones sociales, étnicas, económicas, educativas y discapacidad. Las transformaciones generadas a partir de esta visión de inclusividad se han circunscrito en tres importantes dimensiones: filosófico, normativo y educativo.

En el caso de Costa Rica se han tomado decisiones importantes en el ámbito legislativo y educativo, como la ley 7600 , para lograr una educación en y para la diversidad. Esta ley se emplaza en el tercer estadio evolutivo de la inclusividad (las reformas integradoras), caracterizado por la integración de los estudiantes con necesidades educativas especiales en los centros educativos, con los servicios de apoyo y adecuaciones tendientes a la equiparación de oportunidades y a garantizar iguales condiciones de acceso y participación. No 
obstante, en la dimensión filosófica aún estamos lejos de alcanzar un cambio que se manifieste tanto en las actitudes y valores de todos los ciudadanos y agentes del proceso educativo como en la praxis inclusiva. En todo caso, el pesimismo hay que dejarlo atrás y se debe siempre aspirar a una participación óptima de todos los educandos en un sistema de calidad y sin discriminaciones para lograr excluir las exclusiones, pues tenemos las herramientas pedagógicas para lograrlo, el apoyo jurídico para cumplirlas y la necesidad social por solucionar.

Este paradigma de la inclusividad se ha sustentado en el reconocimiento de la diversidad, la defensa de los derechos humanos, el fortalecimiento de los valores, el respeto a la diferencia, la dignificación del ser humano, el valor de la interculturalidad y la participación social. Esta visión implica, entonces, una ruptura de la concepción tradicional del mundo, creencias, valores y percepciones, así como una concienciación social y política de la necesidad de este cambio paradigmático para mirar, escuchar, estimar y dignificar las singularidades.

En la segunda parte se proponen algunos desafíos de la ley 7600 para lograr la esencia de esta ley: una igualdad de oportunidades educativas y una educación de calidad. Más hay tareas pendientes que requieren asumir responsabilidades y compromisos, no solo los docentes sino todos los involucrados en el acto educativo, desde las altas jerarquías políticas hasta la comunidad, quienes deben trabajar de manera conjunta e integrada para aprovechar al máximo los recursos disponibles y llegar a consolidar una comunidad escolar y una sociedad democráticas y dignas. Mas no es un asunto meramente discursivo o una exigencia normativa, es un cambio de actitud y de visión del mundo a partir de un proceso de develación de nuestras raíces geográficas, históricas, ideológicas y axiológicas que nos permitan comprender nuestras identidades y diferencias, apre- ciar la invaluable riqueza de la naturaleza humana y brindar una oportunidad social a quienes se encuentran en una situación de desventaja, física o de otra naturaleza.

Finalmente, cabe subrayar que este cambio paradigmático y de consciencia es un proceso lento y holístico, en el cual no es suficiente la implementación esporádica de algunas estrategias pedagógicas, las decisiones aisladas de un determinado gobierno, ni un modelo educativo o teorías psicopedagógicas en boga, debe ser resultado de un proyecto curricular, filosófico, social y político para lograr la construcción de una sociedad inclusiva.

\section{Referencias bibliográficas}

Aguado, M. T. (2000). Diversidad, igualdad, cultura escolar: significado e implicaciones prácticas en la enseñanza secundaria obligatoria. Revista Española de Orientación y Psicopedagogía, 20(11), 187-198.

Bautista, R. (1993). Una escuela para todos: Una integración escolar. En R. Bautista (Comp.), Necesidades educativas especiales ( $2^{\text {a }}$ ed., 31-45). Málaga, España: Ediciones ALJIBE.

Braslavsky, C. (2006). Desafíos de las reformas curriculares frente al imperativo de la cohesión social. Revista Electrónica Iberoamericana sobre Calidad, Eficacia y Cambio en Educación, 2e(4), 43-57. Recuperado el 14 de agosto del 2006 de http:// www.rinace.net/arts/vol4num $2 \mathrm{e} /$ art3.pdf

Cánovas, C. E. (2005). Rupturas de paradigmas educativos en la cotidianidad del aula. Revista Electrónica Iberoamericana sobre Calidad, Eficacia y Cambio en Educación, 1(3, Especial), 598-626. Extraído el 11 de agosto de 2006 de http://www. 
ice.deusto.es/rinace/reice/vol3n1_e/ Canovas.pdf

Contreras, J. (2002, marzo). Educar la mirada... y el oído. Percibir la singularidad y también las posibilidades. Cuadernos de Pedagogía, 311, 61-65.

Costa Rica. Asamblea Legislativa de la República. (1996). Ley 7600. Igualdad de oportunidades para las personas con discapacidad. San José, Costa Rica: Editorial Investigaciones Jurídicas.

Devalle, A. y Vega, V. (1999). Una escuela en y para la diversidad. El entramado de la diversidad ( $2^{\mathrm{a}}$ ed.). Buenos Aires, Argentina: AIQUE.

Díaz, E. M. (2003). Atención a la diversidad: Condiciones de la escuela para todos. Bordón. Revista de Pedagogía, 2(55), 191-203.

Echeita, G. y Sandoval, M. (2002, EneroAbril). Educación inclusiva o educación sin exclusiones. Revista de Educación, 327, 31-48.

Fernández, M. (2002, Marzo). Iguales, libres y responsables. Cuadernos de Pedagogía, 311, 56-60.

Fernández,A. (2003, Septiembre). Educación inclusiva: "enseñar y aprender entre la diversidad”. Revista Digital UMBRAL 2000, 13, p. 1-10. Recuperado el 13 de agosto del 2006 de http://portal. perueduca.edu.pe/basicaespecial/articulos/art01_01-09-06.pdf

García, C. (2003). Segregación, integración e inclusión. Bordón. Revista de Pedagogía, 1(55), 9-26.

Guajardo, E. (1999, Octubre-Diciembre). La inclusión e integración en el mundo. Implicaciones teóricas metodológicas y sociales. Revista de Educar,
11. Recuperado el 1 de julio del 2005 de http://educar.jalisco.gob. $\mathrm{mx} / 11 / 11$ indice.html

Irving, B. A., Hartas, D., Barker, V. y Parker-Jenkins, M. (2000). In pursuit of social justice: Careers guidance provision for muslim girls in England [En búsqueda de la justicia social: Guía profesional para jóvenes musulmanas en Inglaterra]. Revista Española de Orientación y Psicopedagogía, 20(11), 173-186.

Malik, B. (2002). Modelos de orientación $e$ intervención psicopedagógica. Proyecto Docente. Madrid, España: Inédito.

Manucci, M. (2004, Abril-Mayo). La gestión de la incertidumbre. El desafío de liderazgo en contextos inestables. Revista Electrónica Razón y Palabra, 38. Recuperado el 4 de febrero del 2005 de: http://www.cem.itesm.mx/ dacs/publicaciones/logos/anteriores/ n38/mmanucci.html

Ministerio de Educación Pública [MEP]. (1997/1998). Políticas, normativa y procedimientos para el acceso a la educación de los estudiantes con necesidades educativas especiales ( $1^{\mathrm{a}}$ Reimpresión). San José, Costa Rica: Autor.

Parrilla, A. (2002, Enero-Abril). Acerca del origen y sentido de la educación inclusiva. Revista de Educación, 327, 11-29.

Palmero, M. C. (2000). Diversidad y educación. Contribuciones científicas y tendencias actuales de investigación en historia de la educación especial. Revista de Ciencias de la Educación, 184, 247-266.

Peralta, F. (2001). La inclusión: ¿Una alternativa al modelo de escuela 
integradora y comprensiva en España? Revista de Ciencias de la Educación, 186, 183-196.

Pérez, J. y Prieto, M. D. (1999). Más allá de la integración, hacia la escuela inclusiva. Murcia, España: Publicaciones de la Universidad de Murcia.

Richardson, R. y Wood, A. (1999/2000). Inclusive schools, society [Escuelas inclusivas, sociedad]. Londres, Inglaterra: Trentham Books.

Rivero, J. (2005). Políticas educativas y exclusión: sus límites y complejidad. Revista Electrónica Iberoamericana sobre Calidad, Eficacia y Cambio en Educación, 3, 33-41. Recuperado el 11 de agosto del 2006 de http://www. rinace.net/arts/vol3num2/art3.pdf
Sandoval, M., López, M. L., Miquel, E., Durán, D., Giné, C. y Echeita, G. (2002). Index for inclusion. Una guía para la evaluación y mejora de la educación inclusiva. Contextos educativos: Revista de Educación, 5, $227-$ 238. Recuperado el 13 de agosto del 2006 de http://www.dialnet.unirioja. es

Sanz, R. (2001). Orientación psicopedagógica y calidad educativa. Madrid, Psicología Pirámide.

Yanes, J. (2008). Sociedad, Ciencia y Enseñanza Superior. Firmas 2007. Reflexiones multitemáticas de filósofos de la Información y el Conocimiento. Recuperado el 27 de mayo del 2008 de: http://static.scribd. com/docs/i05yr3cja46qx.pdf 
